\title{
KOPULATIONS-KULISSEN: \\ ERGEBNISSE UND FORSCHUNGSPERSPEKTIVEN EINER EXPLORATIVEN STUdiE ZU SELEKTIONS- UND \\ NUTZUNGSBEDINGUNGEN VON POPMUSIK IN \\ EROTISCHEN UND SEXUELLEN KONTEXTEN
}

\author{
Michael Ahlers, Christoph Jacke
}

\section{Einleitung}

»(Mindestens) drei Dinge braucht man für einen erotischen Abend: Einen Partner, einen intimen Ort - und die richtige Musik. Denn nichts kann einen prickelnden Moment so zerstören wie die falsche Beschallung zum eigentlich richtigen Zeitpunkt. Doch wenn Marvin Gayes `Sexual Healing`, Barry Whites >Can't Get Enough Of Your Love Ross ein Zusammensein musikalisch untermalen, kann nichts mehr schief gehen« (Pressetext RTL 2009).

Die von Oliver Geissen moderierte »Chart-Show« auf RTL wählt für ihre Folgen bestimmte Mottos aus. Nach den besten Männer-, Frauen-, Dancefloor-, Latin- und Herzschmerz-Songs wurden am 5. Juni 2009 die »erfolgreichsten erotischen Hits aller Zeiten« aufgeboten. Das Zitat freilich deutet in eine Richtung, die den ebenso in der Ankündigung zur Sendung erwähnten Erfolg nicht in monetärer, sondern in sexuell-erotischer Hinsicht versteht: den erfolgreichen Einsatz von Popmusik in erotisch saufgeladenen Alltagssituationen - und erfolgreich kann hier bekanntlich je nach Perspektive und Motivation unterschiedlich definiert sein.

Erotische Popmusik hat offen(hör)bar nicht nur einen Einfluss auf unsere zwischenmenschliche Kommunikation, sondern es gibt mittlerweile einen eigenen Markt mit Ratgebern, Fernsehsendungen und unzähligen Compilations. Doch ganz so einfach und verkürzt, wie es uns eben jene Medienangebote inklusive der oben genannten drei Zutaten für einen perfekten sprickelnden< Abend aus dem Ankündigungstext für die Chart-Show suggerie- 
ren, scheint es doch nicht zu sein zwischen zwei Menschen, die sich anhand von Popmusik in erotische Stimmung versetzen und Voraussetzungen für sexuelle Handlungen schaffen wollen. Oder um es weniger euphorisch als im kommerziellen Boulevardfernsehen und distanziert-nüchterner zu sagen:

»Es erhebt sich die Frage, inwiefern Lustempfindungen oder Zustände der Erregung beim Erleben von Musik und Sexualität miteinander verwandt sind und unter welchen Voraussetzungen Musik als ein Element der nonverbalen Kommunikation erotisierend wirken, die Bereitschaft zu sexuellen Handlungen in irgendeiner Weise regulieren kann« (Kreutz 1997: 293).

Insbesondere die Studien von Gunter Kreutz (1997, 2002, 2008) machen die Schwierigkeit der Erforschung des Zusammenhangs von Popmusik und sexuellen Handlungen deutlich. Noch komplizierter wird es, wenn man darüber hinaus sowohl die in dieses »Thema Nr. 1« gehörigen Unterthemen sondiert als auch den suchenden Blick in die dazugehörigen Forschungen vor allem aus Musik- und Medienkulturwissenschaft wirft. Im Rahmen der Pilotstudie eröffneten sich dabei Spannungsfelder zwischen akademischen Musik- und Medienwirkungs- bzw. -nutzungsanalysen sowie Fragen der Musikselektion, die sich jedoch erstaunlich wenig mit einem derart naheliegenden Feld wie »Pop und Sex« und den Bereichen der »süßesten Emotionen« (Ben-Ze'ev 2009: 213) von romantischer Liebe und vor allem sexuellem Begehren beschäftigt haben.

\section{Forschungsstand und -optionen}

Da das Themengebiet »Pop und Sex « zum einen bislang so wenig und zum anderen äußerst heterogen erforscht ist, soll eine Rasterung einen ersten Überblick v.a. über $\mathrm{Ab}$ - und Eingrenzungen geben und somit die unterschiedlichsten Forschungszweige in einen übergeordneten Zusammenhang bringen. Dabei interessieren sowohl durch Musik mitgestaltete erotische als auch sexuelle Situationen. Erotik und Musik fungieren als symbiotische Mechanismen im Sinne des Soziologen Niklas Luhmann, die »erwartungsgemäß vollziehbare organische Prozesse bezeichnen (Luhmann 1994: 31) und die neben sprachlicher Kommunikation »als Hilfsmittel [...] und als eigenständige Sinnübermittlung « (Luhmann 2008: 12) dienen und Anschlusskommunikationen und -handlungen wahrscheinlicher machen. Die Anbahnung der sexuellen Situation, die durch eben solche kommunikativen Prozesse vollzogen wird, welche Begehrlichkeiten wecken sowie Spannung erzeugen sollen und ein Spiel um Ver- und Enthüllungen, um Nicht-Erfüllung und Erfüllung 
(im Gegensatz zu Pornographie) bedeuten (vgl. Silverstone 2007: 98-117), nennen wir Erotik. ${ }^{1}$

Sofern zu den Themenschwerpunkten überhaupt wissenschaftliche Literatur gefunden werden konnte, wird diese schlaglichtartig und exemplarisch genannt und auf ihre Anwendbarkeit für das eigene Vorhaben überprüft.

\subsection{Annäherungen: \\ Sex in Pop-Song-Titeln, Lyrics, Sounds und Visuals}

Popkultur und ihr Nukleus Popmusik sind offensichtlich stark visuelle Kulturen. Das liegt schlichtweg daran, dass vor allem die Popmusik auf dem Markt der Aufmerksamkeitsökonomie produziert, distribuiert, rezipiert und weiterverarbeitet wird. Klar scheint nicht nur durch Casting-Shows und Musikvideos, dass Popmusik visualisiert werden muss und dass dementsprechend bei Pop und Sex auch sehr schnell an naheliegende Erotisierungen der Musik etwa in den Song-Titeln, den Lyrics, den Platten- oder CD-Covern gedacht werden muss. Die Performances (z.B. Auftritte, Tanz) und Images spielen eine große Rolle. Dabei reicht das Spektrum der Erotisierung von Liebe über Lagerfeuer und Kuscheln bis zu knallhartem Sex oder seiner Andeutung. In der Literatur finden sich dazu vor allem essayistische und fallbeispielhafte Beobachtungen wie etwa von Carmen Wulf (2008), Peter Kemper (2005), Diedrich Diederichsen $(1985,2006)$ oder in der Analyse des Jugendpädagogen Jeffrey Jensen Arnett zu »Sex in Teens' Music and Music Videos« (2002). Aber auch im Sound, in der Instrumentierung, in Rhythmus und Tempo können erotisierende Momente erkannt werden, wie es überblicksartig etwa Alf Gabrielsson und Erik Lindström (2001) für den Einfluss musikalischer Strukturen auf emotionalen Ausdruck herausgearbeitet haben. Allerdings beziehen sich die Autoren hier weder explizit auf aktuelle Popmusik noch auf erotische Emotionen.

\subsection{Abgrenzungen: Pornographie, Zensur, Tabus}

Ebenso naheliegend als Themenschwerpunkt für den Bereich Popmusik und Sex erscheinen, anknüpfend an die Überlegungen der Bedeutung vor allem visueller Elemente für die Erotisierung der Musik, normative oder moralische Brüche von Regeln und Tabus. So spielen Popmusiker zur Konstruktion

1 Vgl. zu Erotik und Liebe in der populären Musik den Beitrag von Dietrich Helms in diesem Band und Helms 2009. Vgl. zu Musik und Kommunikation einführend Jacke 2008, 2009 sowie grundlegend weiterhin Casimir 1991 und Großmann 1991. 
ihrer Images auf allen gerade genannten Ebenen mit dem kontrollierten Regelbruch. Wie eine Konstante ziehen sich Pornographie, Zensur und Tabubruch durch die Geschichte(n) der Popmusik. In den Forschungen finden wir hierzu etwa die Zensur-Studien und Ausstellungen von Roland Seim und Josef Spiegel (2004), eine aktuelle medienwirtschaftswissenschaftliche Studie von Andreas Meier (2009) über den Zusammenhang zwischen gezielten Tabubrüchen und dem Käuferverhalten, die pop-politisch motivierten Diskussionen im Themenschwerpunkt Sex der Zeitschrift Testcard - Beiträge zur Popgeschichte (Büsser et al. 2008) ${ }^{2}$ oder auch die kurze Analyse von Diedrich Diederichsen (2006) zum Verhältnis von Pornographie und Popkultur in Texte zur Kunst.

\subsection{Eingrenzungen 1: Fühlen, Denken, Handeln (Hirnforschung, Wirklichkeiten und Emotionen)}

»Die Hirnforschung, wie sie heute betrieben wird, ist leider reine Repräsentationsforschung. Es wird >nur< gesucht, wo welche Funktion im Gehirn verankert ist « (Martin Hirsch, zit. n. Kim 2009: 24).

Das Zitat des Heisenberg-Enkels und »Eyeplorer«-Gründers Martin Hirsch verdeutlicht eines der großen Probleme der Hirnforschungen der letzten Jahrzehnte: Oftmals bekommt der Laie den Eindruck, es ginge allein um das Suchen nach Orten im Gehirn, die für bestimmte Funktionen zuständig sind. Jenseits solcher für medizinische Zwecke wichtigen Lokalisierungsbestrebungen fallen für den Bereich Pop und Sex v.a. die eher konstruktivistisch orientierten Untersuchungen des Verhaltensphysiologen Gerhard Roth (1996, 2003, 2003, 2007) und des Neurophysiologen und Hirnforschers Wolf Singer (2006) sowie aktuell des Medienwissenschaftlers Matthias Uhl (2009) auf. Vor allem Gerhard Roths große Studie zur Verhaltenssteuerung unserer Emotionen durch das Gehirn (2007) ist dabei von Nutzen, da sie zum einen philosophisch und kulturwissenschaftlich anwendbar erscheint (insbesondere für den Bereich der Gedächtnis- und Erinnerungsforschungen im Rahmen von Pop und $\operatorname{Sex}^{3}$ ) und zum anderen die erwartbaren Schwierigkeiten bei der Erforschung von Zusammenhängen aus Kultur, Musik, Medien, Wahrnehmung, Kognition, Verhalten und Kommunikation sehr deutlich beschreibt (vgl. ebd.: 285-377). Oder um es in den Worten von John A. Sloboda und

2 Dabei geht es um Verschiebungen von Tabugrenzen ebenso wie um Gender, gleichgeschlechtliche Pornographie, Marktwirtschaft und Sexualität, Karikierung von Pornoklischees u.v.m.

3 Popkultur/Popmusik und Gedächtnis- und Erinnerungsforschung stehen erst ganz am Anfang. Vgl. zu einem Forschungsprogramm Jacke/Zierold 2008, 2009. 
Susan A. O'Neill (2001: 419) auf den problematischen, erkenntnistheoretischen Punkt zu bringen: »Our working assumption has been that music has different emotional functions in different contexts. « Wenn zudem mit dem israelischen Philosophen Aaron Ben-Ze'ev (2009: 13) Emotionen als instabil, intensiv, parteilich und kurzlebig skizziert werden können, zeigt sich hier die ganze Komplexität der Analyse des prozessualen Zusammenhangs von Popmusik, Medien, Emotion und Erotik. Dass die Beziehungen zwischen divergierenden Kontexten und Funktionen aber keinesfalls vollkommen beliebig sind, belegen die Studien von Roth und auch Kreutz. Kreutz zeigt sich sensibel gegenüber neurowissenschaftlichen Ansätzen zur Emotionsforschung (vgl. etwa Kreutz 2008: 552-554) und konstatiert zudem: »Wenn aber die Wahrnehmung von Musik von vorhandenen oder nicht vorhandenen Denkstrukturen abhängt, so die Überzeugung, dann sind auch ihre emotionalen Wirkungen erlernt und nicht unmittelbar« (ebd.: 549). Wichtig für die eigenen Studien sind aus dem Rahmen medienkulturwissenschaftlich ankoppelbarer Hirnforschung die Betonung sowohl der jeweils individuellen Wahrnehmung und Wirklichkeitskonstruktion als auch der sozialen Kontexte und autobiographischen Erfahrungswelten speziell für Emotionen und Erinnerungen.

\subsection{Eingrenzungen 2: Kultur, Medien und Emotionen}

Die Überlegungen von Roth, Kreutz und auch Sloboda/O'Neill vernachlässigen allerdings zwei für das Forschungsvorhaben fundamentale Besonderheiten: Die schon erwähnte Bedeutung der medialen Vermittlung von Popmusik sowie eben die Besonderheiten der Popmusik selbst. Wo Roth grundlegend neurowissenschaftlich arbeitet und die Spezifika medialen Wahrnehmens nicht berücksichtigt, da setzen sich Kreutz und die meisten der Beiträge in Juslin/Sloboda (2001) nicht mit aktueller Popmusik auseinander. Daher wurden aus dem ebenso weiten Feld der medienkultur- und kommunikationswissenschaftlichen Forschungen diejenigen Überlegungen herausgesucht, welche medienkulturelle Kontexte, Emotionen und Nutzen/Wirkungen ankoppelbar erscheinen lassen. So sind die grundlegenden Ausführungen zu Medien und Emotionen etwa bei Knut Hickethier (2007), Siegfried J. Schmidt (2005), Roger Silverstone (2007: 98-117) sowie Clemens Schwender und Franz Schwab (2007) hilfreich, weil sie die Beeinflussung von Emotionen durch mediale bzw. medienkulturelle Ebenen berücksichtigen und problematisieren. Schmidt differenziert ganz grundlegend $\gg z$ wischen der Emotionalität der Kommunikation bzw. genauer der Kommunikationspartner in konkreten Situationen und der Thematisierung von Emotionen in der Kom- 
munikation « (Schmidt 2005: 25), und Hickethier betont die thematische Anbindung, die Emotionen benötigen, erst Recht, so dürfen wir ergänzen, im Fall von Popmusik: »Emotionen müssen sich mit einem Sachverhalt verbinden, damit sie wirksam werden« (Hickethier 2007: 105). Silverstone (2007) beschreibt erotisches Vergnügen in deutlich medialisierten Welten. Zusätzlich ist für den Zusammenhang aus Kultur, Medien und Emotion insbesondere für den Bereich der Untersuchung von Rezeptionsmotiven und -strategien die Berücksichtigung von Medienwirkungs- und -nutzenansätzen aus der eher kommunikationswissenschaftlichen Forschung sinnvoll (z.B. Uses \& Gratifications, Bias- und allgemeine Medienwirkungsforschung), die allerdings zumeist emotionale Aspekte vernachlässigen (vgl. einführend Merten 1999 und Schenk 1987).

\subsection{Eingrenzungen 3: (Pop-)Musik und Emotionen}

Das letzte, für die theoretische Rahmung der Studie wichtige Forschungsfeld ist der Bereich der musikpsychologischen und -soziologischen Emotionsforschung. Hier halfen uns vor allem allgemeine Überlegungen zu Musik und Emotion von Walter Ludwig Bühl (2004: 135-183), Tia DeNora (1997, 1999, 2001), Leonard B. Meyer (2001), Patrik N. Juslin und John A. Sloboda (2001) bzw. Sloboda und Susan A. O'Neill (2001) sowie von Sloboda, Alexandra Lamont und Alinka Greasly (2009) zur Auswahl von Musik. Dazu kommen vor allem die schon mehrfach genannten Analysen von Gunter Kreutz und eine umfassende Studie von Holger Schramm zum »Mood Management «, also zur Stimmungsregulierung durch Musik, die mal eher schlechte Stimmung ausgleichen (Kompensationsprinzip), mal gute Stimmung aufrecht erhalten oder verstärken soll (Iso-Prinzip, vgl. Schramm 2005: 77-83). Was diesen Studien fast allen abgeht - Kreutz und Schramm bilden Ausnahmen, weswegen diese auch vermehrt zu Rate gezogen wurden -, sind sowohl die Bedeutung der Medien für musikalische Emotionserzeugung als auch erneut die Berücksichtigung aktueller Popmusik. Empirische Studien sind kaum zu finden, aber man ist sich einig, dass etwa Musik als Stimmungsregulator gezielt eingesetzt wird: »Individuals have considerable awareness of how they can use music for personal functions such as mood management « (Sloboda/Lamont/ Greasley 2009: 435). Innerhalb ihrer Kategorisierung zu Funktionen selbst ausgewählter Musik vernachlässigen allerdings auch diese Autoren erotische und sexuelle Situationen vollkommen.

Es bleibt festzuhalten, dass zum großen Zusammenhang von Popmusik, Medien und Sex auffallend wenig konkrete Forschungsliteratur zu finden ist - und zwar sowohl im deutsch- als auch im englischsprachigen Raum. 
Auffallend ist, dass praktisch keine der Studien den Einsatz von Popmusik, medial vermittelt, für erotische oder sexuelle Alltagssituationen analysiert hat. So findet sich auch im Handbuch Musik und Medien (Schramm 2009) weder ein Artikel noch ein Sachregister-Eintrag zur Wechselbeziehung aus Pop, Medien und Sex. Dennoch wurde sich bemüht, aus der Forschungsliteratur zentrale Problemfelder und Fragestellungen zu generieren. Klar scheint also neben einem eklatanten Forschungsdesiderat derzeit lediglich gleichzeitig die Bedeutung von Musik und Medien für Emotionen:

»Music is a cultural material (as is language) that provides a kind of semiotic and affective spower which individuals use in the social construction of emotional feeling and displays. As such, the impact of music on emotion is not direct but interdependent on the situations in which it is heard « (Sloboda/O'Neill 2001: 415).

\section{Problemfelder und Fragenstellungen: Möglichkeiten und Schwierigkeiten}

Aus der Sichtung ergaben sich folgende Problemfelder und Fragestellungen, die sich aus den Vorüberlegungen herausfiltern und zu Hauptthemenblöcken und Seitenthemen konzentrieren lassen, die aufgrund ihrer Häufigkeit, Intensität, bisherigen wissenschaftlichen Beobachtung und empirischen Überprüfbarkeit ausgewählt wurden:

- Qualifikationen von Popmusik für erotische Alltagsituationen - das Erotische an/von Musik: Welche Aspekte von Popmusik spielen eine entscheidende Rolle für deren Auswahl in erotischen Alltagssituationen? Inwiefern wird Popmusik bei welchen Menschen »als lebensgestaltende Funktion « (Kreutz 1997: 295) auch im sexuellen Bereich verwendet?

- Motive, Absichten und Pläne: Für welche erotisch aufgeladenen Situationen wird welche Musik wie eingesetzt - so sie es denn wird (und das auch noch zugegeben wird)? Wie soll dadurch Einfluss auf wen (das Selbst, den Partner, beide, die Situation) genommen werden (vgl. Kreutz 1997: 298)?

- Beziehungen und Rituale: Inwiefern kann Musik als »Zeitzeuge « (Schramm 2005), »Generationsmarker « (Gries 2006), »Erinnerungsanlass« (Zierold 2006) oder sogar »Traditionsstrom« (Assmann 1992) in bestimmten erotischen zwischenmenschlichen Konstellationen fungieren?

- Aktionen und Handlungen: In welcher Phase des eigentlichen Geschlechtsakts wird Musik wie eingesetzt (vgl. Kreutz 1997)? 
Für alle Problemfelder bzw. Themenschwerpunkte soll zudem überprüft werden, ob es Auffälligkeiten oder Regelmäßigkeiten bei den demographischen Daten der Probanden wie Alter, Geschlecht, Beruf zu beobachten gibt, die sich in die theoretischen Vorüberlegungen einpassen oder auch gerade nicht einfügen lassen.

Wie schon erwähnt, wurden zusätzlich einige vermeintlich sekundäre Aspekte aus der Literatur und den Voruntersuchungen generiert, die ebenfalls in die empirische Studie einflossen. So etwa fiel bereits Sloboda/O'Neill (2001: 416) auf, dass Musiker Musik in ihrem Alltag anders erleben und dementsprechend anders einsetzen als Nicht-Musiker. Ferner soll überprüft werden, ob es bestimmte Gruppen von Menschen gibt, die sich in ihren Zuschreibungen von Tabubruch und Pornographie unterscheiden - hierbei scheinen Gender-Aspekte besonders interessant. ${ }^{4}$ Und letztlich sollte der Bereich der medial-kommerzialisierten Aufarbeitung der sexuell-erotischen Auswirkung von Popmusik im Rahmen von Charts (z.B. die genannte »ChartShow « auf RTL), Rankings und Compilations noch einmal genauer betrachtet werden, da in diesem Feld nicht nur kommerzieller Erfolg, sondern auch über das Sammeln, Aufbewahren und Verwalten kurzfristig »Gedächtnisleistungen« (Roth 2007: 153), mittelfristig Erinnerungen und langfristig eine Kanonisierung von Popmusik manifestiert wird. ${ }^{5}$

\section{Die Pilot-Studie »Kopulations-Kulissen«}

\subsection{Organisation und Methodik}

Im Sommersemester 2009 befassten sich ein Bachelor- und ein Masterkurs des Studiengangs »Populäre Musik und Medien« der Universität Paderborn unter Anleitung der Verfasser thematisch und forschungsbegleitend mit den oben benannten Themengebieten. ${ }^{6}$ Im Zentrum der ersten Semesterhälfte stand nach erfolgreicher Literaturanalyse die Konstruktion eines Leitfadens (vgl. Bortz/Döring 2006: 315), der als Gerüst der folgenden qualitativen Da-

4 Vgl. die Beiträge in Büsser et al. 2008 sowie in Eismann 2007 und Reitsamer/ Weinzierl 2006.

5 Vgl. zu popmusikalischen Kanonisierungen Korte 2008, von Appen/Doehring/ Rösing 2008 und zu popkulturellen Erinnerungen und Festschreibungen Jacke/ Zierold 2008, 2009 sowie Jacke/Meinecke 2008.

6 Allen Studentinnen und Studenten der Kurse "Sex Sells Pop - Ein empirisches Forschungsprojekt « und »Popmusik, Emotionen und Erotik« aus dem Sommersemester 2009 sei an dieser Stelle unser Dank ausgesprochen. Besonders hervorzuheben ist Timon Tobias Temps. 
tenerhebung (via halbstrukturierter Leitfaden-Interviews) diente. Die Konstruktion des Leitfadens erfolgte entlang der im vorigen Kapitel ausgeführten Themenschwerpunkte, wobei nach dem Pre-Test noch insgesamt fünf Themenschwerpunkte und zehn Hauptfragen eingesetzt wurden. Die Interviews fanden im privaten und öffentlichen Raum mit einer Gelegenheitsstichprobe statt. Anschließend wurde der Materialkorpus über qualitative Inhaltsanalysen (Mayring 2008) zusammengefasst.

Hieraus konnten anschließend die Konstrukte und Skalen (Auswahl, Einsatz, Qualifikation, Tabubrüche usw.) für das Fragebogen-Instrument operationalisiert und gebildet werden. Die Skalen wurden - diesmal von einer Zufallsstichprobe (Bortz 2005: 86) - über vier- bzw. fünfstufige Likert-Skalen (s.u.) bewertet. Es sollte ein Untersuchungs-Design gewählt werden, in dem sich an eine qualitative Exploration die quantitative Fragebogen-Erhebung und eine abschließende qualitative Überprüfung anschließen (vgl. Flick 2007: 43). Der Forschungsprozess endete letztlich mit der Begutachtung des Testentwurfs, der Itemanalyse, einer ersten beschreibenden und analytischen Auswertung sowie der Interpretation der Ergebnisse (vgl. Bühner 2004: 45, Zöfel 2008: 12).

\subsection{Beschreibung und Auswertung der qualitativen Vorstudie}

Nach einer ersten »Probe-Befragung «, die vor allem der Identifizierung von allgemeinen Interviewfehlern und der Optimierung der Interviewmethode bzw. der -fragen (vgl. Helfferich 2005) diente, konnten insgesamt 28 qualitative Interviews geführt werden. Die Probanden der Vorstudie waren zwischen 19 und 66 Jahren $(M=32,82$ Jahre) alt und annähernd ausgeglichen auf die Geschlechter (12 weiblich/16 männlich) verteilt. 10 Teilnehmende gaben als höchsten Bildungsabschluss das Abitur an, zwei Personen verfügten über ein Staatsexamen, drei über einen Diplom-Abschluss.

Der verwendete Leitfaden-Katalog beinhaltete zunächst Gebiete aus dem Bereich Alltagswissen und Klischeebildungen, die im quantitativen Forschungsprozess jedoch nicht weiter verfolgt wurden. So entstanden beispielsweise Fragen zur erotisierenden Kraft von Musikinstrumenten und inwieweit hierbei eher der Klang oder das Aussehen eine Rolle spielen. Es ist bemerkenswert, dass hierauf 16 der 28 Probanden antworteten, dass der Klang für sie wichtiger sei. Die konkrete Nennung eines »erotischen Instruments« führte zu einer Rangliste, auf der Gitarre und Streichinstrumente mit acht Nennungen vorn platziert sind, gefolgt von der Stimme, dem Klavier und dem Saxofon. Aussagen zur Entstehung der eigenen, mental oder 
physisch vorhandenen, »erotischen Plattensammlung « und existenten Ritualisierungen im Umgang mit Musik und Erotik waren ebenfalls aufschlussreich:

»Na ja, man sucht halt, und man findet immer. Man guckt im Internet oder im Fernsehen, was da so immer in den Top-Listen ist, was gut ist, und dann guckt man. Oder was einem selber halt gefällt, was man über die Jahre sich so halt aneignet« (männlich, 19 Jahre, Nebenjob bei Sicherheitsdienst).

»Über die großartigen technischen Möglichkeiten, die uns mittlerweile gegeben sind. So dass man generell vielleicht Musik laufen hat, bei der man merkt - in erotischen Situationen oder beim Sex - welche Musik funktioniert oder welche nicht funktioniert und dies dann abgespeichert hat und dann wieder der neuen Playlist zugeordnet hat, um nicht mehr den Umstand zu haben, ein Lied weiter zu drücken, wenn man vielleicht gerade mit anderen Dingen beschäftigt ist « (männlich, 30 Jahre, Lehrer).

Man kann hier ablesen, wie sehr die mediale Rezeption einerseits und die Verbindung zwischen funktionalen Entscheidungen und technischen Entwicklungen der vergangenen zehn Jahre andererseits Einfluss auf individuelle Auswahlprozesse nehmen können.

Zur Konstruktion der Skalen des quantitativen Teils der Studie wurden weiterhin musikalische Parameter erfasst, die laut Aussage der Probanden für eine erotisierende Wirkung von Musik wichtig sein können. Hierbei ergab sich, dass der Parameter »Tempo« (18 Nennungen) als wichtigster Faktor angesehen wird. Diese Auszählung basiert unter anderem auf Aussagen wie: »Ja, wenn's jetzt Schmusesongs sind, die sind ja meistens immer langsamer vom Rhythmus, und das ist es vielleicht, was einen zum Sich-Fallen-Lassen bringt « (weiblich, 44 Jahre, kaufmännische Angestellte), auch wenn hier offensichtlich Rhythmus synonym mit Tempo benutzt wird. Aussagen wie diese finden sich im Material häufig und sie unterstützen die von Kreutz (1997: 298) beschriebene Verhaltensregulation in Bezug auf das Selbst sehr deutlich. Zusätzlich wurde die Stimme als wichtiger Faktor (16 Nennungen) genannt, was gerade durch neuere Untersuchungen zur Rolle und Funktion der Stimme in der Popmusik (vgl. Pfleiderer 2009) erklärbar wird. Weitere Parameter sind Lautstärke, Rhythmus, Instrumentierung, »der Interpret selbst « und die Melodie eines Songs.

Ebenso konnten die Skalen zur Erfassung erotisierender bzw. als störend empfundener Musikstile aus dem qualitativen Material heraus gebildet werden. Hierbei wurde »Popmusik« mit 14 Nennungen am häufigsten als »erotisch« bezeichnet und »Heavy Metal« als eher störend beschrieben. Auch wenn die Festlegung von Genres dabei mitunter zu Missverständnissen und 
Auslassungen führte, erwies sich die so generierte Liste als für den weiteren Verlauf der Untersuchungen fruchtbar.

Weiterhin konnten die Skalen zur (bewussten oder unbewussten) Auswahl von Musik gebildet werden, da die Probanden hierzu sehr offen und bereitwillig beitrugen: "Ich setze Musik in erotischen Situationen manchmal schon ganz bewusst ein. Also um zum Beispiel Atmosphäre oder um eine gewisse Anregung zu schaffen« (männlich, 21 Jahre, Ausbildung zum Konstruktionsmechaniker); »Schon noch bewusst. Wir haben eine Suchmaschine. Die spuckt uns dann die Musik aus. Sie muss ruhig sein. Schon auch ein bisschen melancholisch« (weiblich, 35 Jahre, Zahnarzthelferin); oder: »Das ist ganz unterschiedlich, manchmal gar nicht, manchmal um vielleicht ein paar Geräusche zu überdecken, manchmal aber auch, weil es mir oder meiner Freundin gefällt und es nette Atmosphäre schafft« (männlich, 22 Jahre, Ausbildung zum Mechatroniker). Interessant ist hieran abzulesen, wie die Faktoren des »Mood Managements" (vgl. die Grafik von Bullerjahn in Schramm 2005: 53) einerseits, die partnerschaftliche »Synchronisation « (vgl. Kreutz 1997: 298) und Erzeugung von Intimität andererseits offenbar mit in die individuelle Auswahl hineinspielen. ${ }^{7}$

Letztlich wurden die Bereiche möglicher Tabubrüche und der Pornographie im Zusammenhang mit Popmusik als potenzieller Seitenaspekt der Studie erfasst. Es wurden die nach Einschätzung der Probanden wichtigsten $\mathrm{Pa}$ rameter eines solchen »pornographischen Potenzials« für Musik abgefragt. Es wurde hierbei klar, dass vor allem Rap-Musik in den Augen vieler Probanden aufgrund der expliziten und misogynen Texte in der Lage ist, Grenzen zu überschreiten oder Tabus zu brechen:

»Es gibt natürlich in Kombination mit Musikvideos sehr, sehr, äh, erotische Sachen die, die, dann, ja [Pause] schon fast Softporno sind, äh, oder in die Richtung gehen. [...] Ja es gibt da natürlich auch noch so Videos, die für mich mit Erotik nichts mehr zu tun haben, das sind da so, ja, äh, Rap oder, oder HipHop oder so, wo Frauen einfach ja, keine Ahnung. Ja, so, die einfach nur noch verachtend sind « (männlich, 24 Jahre, Hotelfachkraft).

Weitere Faktoren potenzieller Tabubrüche wurden in den Bereichen »Outfits« oder »übertriebene Darstellung von Sexualität« in den Auftritten und Videos der Musikerinnen und Musiker beschrieben: »Ja auf jeden Fall! >Dirrty< oder >Super Bitch< von Christina Aguilera: sie übertreibt es einfach, diese Art von Songs ist eher billig, hat nichts Erotisches an sich« (männlich, 29 Jahre, Verkäufer im Außendienst). Der überwiegende Teil der Partizipie-

7 Vgl. allgemein zum Zusammenhang von Musikauswahl und erotischen Handlungen DeNora 1997 und Kreutz 2002, 2008. 
renden verstand die Frage dahingehend richtig, als dass »Musik selbst «bzw. ihr Sound von nur einem Probanden als potenziell pornographisch beschrieben wurde. ${ }^{8}$

\subsection{Beschreibung der quantitativen Pilotstudie}

Die Fragebogen-Erhebung wurde als »Papier und Bleistift-Untersuchung « durchgeführt und durch die Interviewenden ausgefüllt. Abgesehen von der Skala »Erwünschtheit«, die aus einer Untersuchung von Gunter Kreutz (1999: 404) stammt, wurden alle Skalen neu gebildet. Die Skalen sind auf nominalem und ordinalem Niveau angesiedelt und als Rating-Skalen (Likert) formuliert. Die Daten wurden im öffentlichen Raum (Bus, Bahn, Einkaufszentren) der Stadt Paderborn, der Region Westfalen und im privaten Bereich anhand einer Zufallsstichprobe erhoben.

Die Stichprobe umfasste 113 Versuchspersonen (Vpn) zwischen 18 und 69 Jahren $(M=30,97$ Jahre, $S D=1,2)$, verteilte sich aber zu fast zwei Dritteln $(65,4 \%)$ auf den Altersbereich 18 bis 29 Jahre, zu jeweils knapp $10 \%$ auf die Altersbereiche 30 bis 39 Jahre und 40 bis 49 Jahre, sowie zu je $5 \%$ auf die Altersbereiche 50 bis 59 und 60 bis 69 Jahre. Sie bestand zu 41,6\% aus weiblichen und zu 58,4\% aus männlichen Teilnehmenden, wobei Studenten $(36,3 \%)$, Angestellte $(26,5 \%)$ und Selbständige $(10,6 \%)$ die größten Gruppen innerhalb der Angaben zur Beschäftigungs- bzw. Ausbildungssituation bildeten. Etwa die Hälfte der Vpn (51,3\%) gab an, ein Instrument zu spielen.

Die Analyse der Skalen zeigt in zwei Fällen gravierende Probleme im Bereich der Reliabilität: Während sechs Skalen mit Werten für Cronbachs $a=$ .69 und $a=.8 \mathrm{im}$ mittleren bis unteren Bereich für explorative Studien liegen, fallen zwei Skalen aufgrund ihrer geringen Reliabilität prinzipiell für eine statistisch abgesicherte Interpretation ihrer Werte aus: Die durchgeführte Faktorenanalyse (Hauptkomponenten-Analyse mit Varimax-Rotation) zeigt, dass innerhalb der Skala »Musikalische Parameter« offenbar zwei Komponenten und in der Skala »Beziehungen « drei Komponenten extrahiert werden können, auf welche die einzelnen Items laden. Es sind hier Probleme innerhalb der Konstrukte zu erkennen, die in der Neubildung mehrerer Subskalen für die benannten Bereiche resultieren werden.

8 Hieran wird die Bedeutung der bereits in Abschnitt 2 erwähnten subjektabhängigen und gleichermaßen sozial orientierten Wirklichkeitskonstruktion (vgl. Roth 2007, Schmidt 2005, Singer 2006) bzw. die Bedeutung dieser Faktoren für die Funktionalisierung von Musik klar (vgl. Dollase 1974, Dollase et al. 1986; Lehmann 1994). 
Aufgrund der Probleme im Bereich der Reliabilität und der nicht vorhandenen Normalverteilung innerhalb der Stichprobe resultiert für die folgende Datenauswertung, dass zunächst deskriptive und nicht-parametrische Verfahren zum Einsatz kommen können. Da die vorliegenden Studie stark explorativen Charakter hat und das Ziel der Forschung unter anderem in der Initiierung einer transdisziplinär angebundenen Diskussion besteht, werden im weiteren Verlauf mitunter bewusst Trends und Ergebnisse auf Variablenebene beschrieben, um so in Folgestudien und über Repliken auf diesen ersten Vorschlag hoffentlich in einen produktiven Diskurs über die Messbarkeit und Sinnhaftigkeit bestimmter Konstrukte einsteigen zu können.

\subsection{Auswertung der Stichprobe}

Musik wird innerhalb der erfassten Gruppe vorwiegend im privaten Umfeld gehört. Am häufigsten wurde hier der private Bereich »Zuhause« $(M=3,25$, vierstufige Likert-Skala) genannt, neben öffentlichen bzw. teil-öffentlichen Bereichen wie »beim Autofahren « $(M=3,61)$ und »Bar/Disco $(M=3,35)$. Mehr als zwei Drittel der Befragten gab an, selten oder gar keine Musik bei der Arbeit zu hören. 65,5 Prozent der Stichprobe hören nach eigenen Angaben mindestens drei bis fünf Stunden pro Tag Musik. Nur ein Drittel der Vpn hört drei Stunden oder weniger Musik pro Tag. Insgesamt sind die Teilnehmer der Stichprobe damit zu zwei Dritteln als Intensivhörer zu bezeichnen, vergleicht man die Werte mit den Ergebnissen aus der Verbrauchs- und Medienanalyse 2010 (Freizeitaktivitäten: 1-3) oder der Studie Massenkommunikation (vgl. Fritz/Klingler 2006). Lediglich Jugendliche zwischen 12 und 19 Jahren konsumieren im Bundesdurchschnitt am Tag noch länger Musik (vgl. Medienpädagogischer Forschungsverbund Südwest 2008). 20 Personen der Stichprobe können gar als »Musikfreaks « bezeichnet werden, da sie alle Aussagen der Skala zur persönlichen Musiknutzung hoch oder sehr hoch unterstützt haben. Diese Teilgruppe ist zu gleichen Teilen auf die Geschlechter verteilt, zu 90 Prozent unter 30 Jahre alt und zu 60 Prozent mit Studierenden und zu 20 Prozent mit Angestellten besetzt.

Mehr als die Hälfte $(58,4 \%)$ der Vpn gab an, Musik bewusst zu hören. Ein Großteil der Befragten gab an, über die bewusste $(80,6 \%)$ bzw. unbewusste $(65,2 \%)$ Musikauswahl ihre Stimmung verbessern zu können. Die Aussagen zur bewussten oder zufälligen Unterstützung ihrer jeweiligen emotionalen Disposition (positiv oder negativ, vgl. Schramm 2005) über die Musikauswahl wurden bei allen Probanden nahezu gleich $(M=3,89$ für »bewusst « und $M=$ 3,79 für »zufällig«, fünfstufige Likert-Skala) bewertet. An dieser Stelle sei auf die in diesem Punkt kontrovers diskutierte »Mood Management«-For- 
schung etwa bei Juslin/Sloboda (2001a: 453ff.), Sloboda/Lamont/Greasley (2009) und Schramm (2005) verwiesen, die das fragile Beziehungsgeflecht zwischen Musik und Emotionen und die mitunter diffizilen und oftmals falsch verstandenen oder falsch verwendeten Begrifflichkeiten und hieraus resultierenden Konstrukte kritisch reflektieren.

Die aus der qualitativen Vorstudie extrahierten Bedingungen für die Entstehung von erotischen und sexuellen Situationen selbst können durch die quantitativen Daten abgesichert und als konsensfähig bezeichnet werden. So stimmen die Probanden den Aussagen, gedämpftes Licht, die »richtige Stimmung «, ein/e erotische/r Partner/in, ein ungestörtes Umfeld und ausreichende Zeit füreinander seien wichtig oder sehr wichtig, zu 66 bis $92,9 \%$ zu.

\section{Qualifikationen von Popmusik für erotische Alltagssituationen}

Anders hingegen fallen die Ergebnisse der Skala zur Erfassung »musikalischer Parameter « erotischer Musik aus, deren Probleme bereits weiter oben thematisiert wurden. Ein langsames Tempo (45,1\%), der Musiker selbst $(48,7 \%)$, ein unterstützender Rhythmus (49,5\%) oder die Stimme des Musikers $(87,6 \%$ [!]) werden von den Befragten als wichtige oder sehr wichtige Faktoren erachtet. Ebenso sind 68,2\% der Vpn der Auffassung, die Musik selbst sollte nicht laut sein. Die Zuschreibung einer Fähigkeit der »Erotisierung « von Situationen fand vor allem bei den Genres Soul (16\%), Lounge $(13,9 \%)$, Jazz $(10,8 \%)$ und R'n'B $(9,7 \%)$ statt. Im umgekehrten Frageblock wird Heavy Metal $(19,4 \%)$, Schlager $(17,6 \%)$, HipHop $(13,5 \%)$ und House/Techno $(9,7 \%)$ hingegen störendes Potenzial zugeschrieben. Diese Aussagen können als eine Mitbegründung des derzeit für fast alle genannten Sparten verfügbaren Marktes »erotischer Liedzusammenstellungen« (s.u.) angesehen werden, bieten sich der Musikindustrie hier doch offensichtlich zahlreiche Hauptund Nischenmärkte. Die Bewertung der Qualifikation von Musikstilen in Abhängigkeit unterschiedlicher situativer und emotionaler Rahmenbedingungen scheint in dieser Stichprobe eher individuell zu sein und deutet auf die von Ben-Ze'ev (2009: 13-84) ausführlich beobachtete sehr starke Parteilichkeit und Instabilität hin.

\section{Motive, Absichten und Pläne}

Die Antworten in der Skala zum Einsatz und der Funktionalisierung von Musik in erotischen Situationen offenbaren, dass Dreiviertel der Vpn angeben, durch ihre Musikauswahl eine erotische Stimmung erzeugen zu wollen. Etwas mehr als die Hälfte $(56,7 \%)$ gibt dabei an, vor allem die Umgebung ausblenden zu wollen. Die pragmatische Angabe, Musik während des Sex vor 
allem zur Überspielung der eigenen Geräusche einzusetzen, wird von 69,1\% der Stichprobe abgelehnt.

\section{Beziehungen und Rituale}

Mehr als die Hälfte $(56,7 \%)$ der Befragten unterstützen die Formulierung, dass ein Lied im eigenen Lebensverlauf erotisierend wirken kann, wenn dieses ursprünglich in einer erotischen Situation gehört wurde. ${ }^{9}$ Während die weiteren Items der Skala zu keinen konkreten Trends führen, lehnt über die Hälfte der Befragten (56,6\%) die Behauptung ab, die »erotische Kraft« von Songs verliere im Laufe einer Beziehung potenziell an Stärke.

\section{Aktionen und Handlungen}

In Bezug auf die Studie von Gunter Kreutz (2002) wurde dessen Fragestellung wiederholt, in welchen Phasen des Kennenlernens und des Liebesspiels selbst Musik erwünscht ist. Es lässt sich bestätigen, dass auch in der vorliegenden Untersuchung knapp zwei Drittel der Probanden gerne beim Flirt, beim Vorspiel und dann wieder beim Nachspiel/Schmusen Musik hören, während des Geschlechtsakts jedoch keinen besonderen Wert darauf legen. Geschlechtsspezifische Unterschiede, wie sie in der ursprünglichen Studie auch erhoben wurden, lassen sich im Datenmaterial nicht bestätigen. Bemerkenswert ist die Tatsache, dass in der vorliegenden Untersuchung unter den männlichen Probanden der Wunsch, auch während des Aktes selbst Musik zu hören, im Mittel vergleichbar mit den Angaben der weiblichen Vpn ist, jene aber deutlich häufiger zustimmen, beim Nachspiel bzw. Schmusen hinterher gerne Musik zu hören:

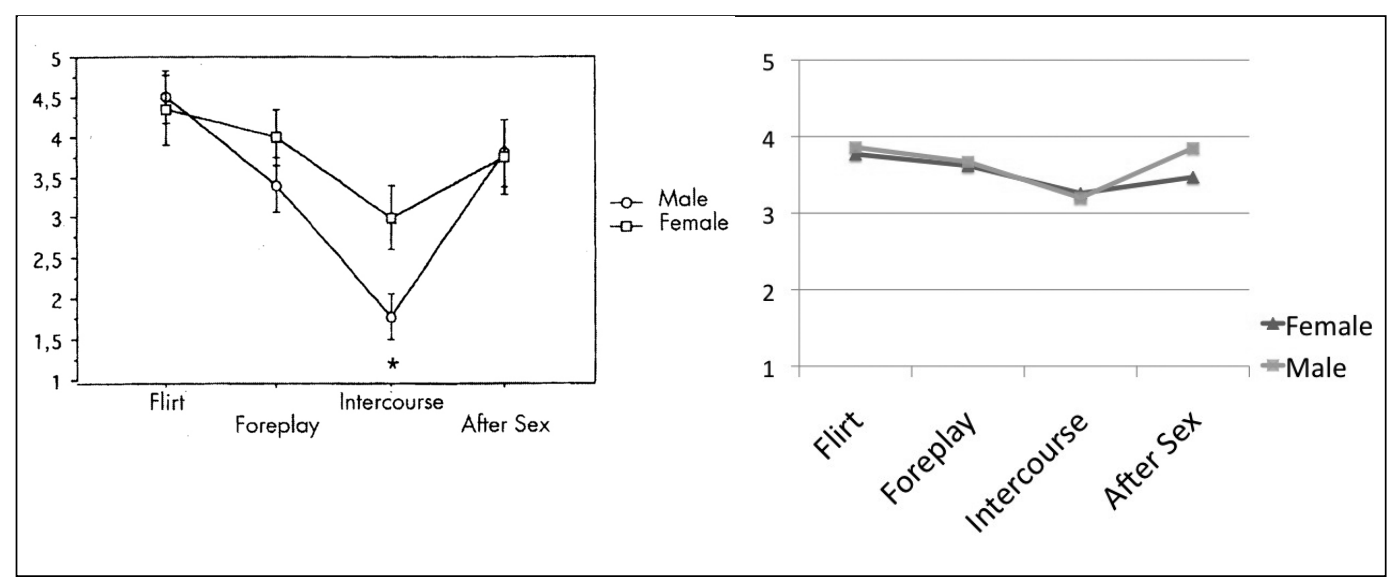

Abbildung 1: Ergebnisse der Originalstudie Kreutz (1999) vs. eigene Ergebnisse

9 Autobiographische Erinnerungen in kollektivem Abgleich scheinen insbesondere in Bezug auf Popmusik eine wichtige Rolle zu spielen (vgl. Jacke/Zierold 2008, 2009). 
Tabubrüche

Bedingt durch die reichhaltige Literatur zu diesem Seiten-Thema (s. o.) und jüngste mediale Inszenierungen wurde der Bereich »Tabubruch und Pornographie « mit aufgenommen. Hierbei zeigt sich, dass die Teilnehmenden des quantitativen Teils vor allem Rap (58 von 77 Nennungen) als potenziell geeignet sehen, Tabus zu brechen, gefolgt von Heavy Metal (11 der 77 Nennungen). Die Items wurden hier ebenfalls aus der Vorstudie gewonnen und zeigen nun, dass für Tabubrüche in den Augen der Befragten ebenfalls vor allem anstößige (44\%), Gewalt verherrlichende (39,8\%) oder Frauen verachtende $(35,4 \%)$ Texte verantwortlich gemacht werden. Obszöne Auftritte und freizügige Videos als Faktoren führten in diesem Punkt nur zu geringer Zustimmung bzw. gar leichter Ablehnung bei einem Teil der Vpn. Die Aussage, der Sound von Musik selbst könne pornographisch sein, wurde von 55\% der Befragten abgelehnt, die restlichen Probanden standen dieser Aussage eher neutral gegenüber. Hier deckt sich die Antworttendenz mit den Ergebnissen der Vorstudie.

\subsection{Ausgewählte Ergebnisse}

Nachfolgend wurden ausgewählte soziodemographische Faktoren wie der Beruf, instrumentale Vorkenntnisse und das Geschlecht mit den Variablen der einzelnen Skalen kreuztabelliert und über den Einsatz von Chi-QuadratTests (exakter Test nach Fisher) untersucht, inwieweit sich hier signifikante Gruppenunterschiede bzgl. der Verteilungen beschreiben lassen. Instrumentale Vorkenntnisse schienen bereits in der Vorstudie zu spezialisierten Antworten zu führen, die ein vermeintlich durch den eigenen Expertisegrad geprägtes Rezeptions- und Funktionalisierungsmuster von Musik vermuten ließen. Auch wenn hierzu bereits im musikpsychologischen Bereich zahlreiche Arbeiten in Bezug auf die spezialisierte Wahrnehmung, Bedeutungs- und Emotionszuschreibung sowie den Einfluss der Performance von Musikern versus Nicht-Musikern vorliegen (vgl. Sloboda/O'Neill 2001, Lehmann 1994) wurden speziell die Bereiche Popmusik und vor allem die Auswahl und der Einsatz von Musik für die Anbahnung und Durchführung sexueller Handlungen bisher kaum beforscht.

\section{Genderaspekte}

Frauen geben in dieser Stichprobe signifikant häufiger an, Musik bewusst und nicht bloß nebenbei zu hören $\left(C h i^{2}=10,7, d f=4, p=.02\right)$. Ebenso geben sie häufiger an, Musikvideos als zu freizügig zu empfinden ( $p=.0016)$. Männer hingegen geben signifikant häufiger an, ihre jeweilige Stimmung zu- 
fällig durch die getroffene Musikauswahl zu unterstützen $\left(\mathrm{Chi}^{2}=9,1, d f=4\right.$, $p=.04)$ und durch sie in erotischen Situationen die Umgebung ausblenden zu wollen $\left(C h i^{2}=8,9, d f=4, p=.04\right)$. Weiterhin wählen Männer nach eigenen Angaben hoch signifikant häufiger die Musik in einer Beziehung aus als Frauen $\left(C h i^{2}=14,5, d f=4, p<.01\right)$.

\section{Berufe}

Innerhalb der Untersuchung lassen die beiden Gruppen der studentischen und selbständigen Teilnehmerinnen und Teilnehmer spezifische Profile erkennen. Für Angestellte konnten keine signifikanten Unterschiede erfasst werden.

Für den Bereich der Stimmungsregulierung geben Studierende hoch signifikant häufiger an, durch das Musikhören ihre Stimmung bewusst zu unterstützen $\left(\mathrm{Chi}^{2}=10,6, d f=4, p=.02\right)$ sowie hierdurch in der Lage zu sein, negative Stimmungen überwinden zu können $\left(C h i^{2}=9,1, d f=4, p=.04\right)$. Sie lehnen es ab, dass der erotische Gehalt eines Liedes im Verlaufe einer Beziehung verloren gehen könne $\left(\mathrm{Chi}^{2}=9,4, d f=3, p=.02\right)$. Innerhalb der Erfassung von Tabubrüchen und pornographischen Merkmalen von Musik sind sie es, die signifikant häufiger der Aussage zustimmen, dass vorwiegend anstößige Texte in der Lage sind, Tabus zu brechen $\left(C h i^{2}=8,4, d f=3, p=\right.$ $.03)$.

Selbständige Teilnehmende lehnen die Aussage signifikant häufiger ab, Musik hauptsächlich nebenbei zu hören $\left(C h i^{2}=9,4, d f=4, p=.04\right)$, und stimmen häufiger zu, sie bewusst zu hören $\left(\mathrm{Chi}^{2}=9,2, d f=4, p=.045\right)$. Innerhalb der Skala zur Erfassung wichtiger Faktoren für die Entstehung erotischer Situationen stimmen sie der Aussage, dass eine richtige Stimmung hierfür wichtig ist, hoch signifikant häufiger zu $\left(C h i^{2}=11,3, d f=4, p=\right.$ .014).

\section{Instrumentalisten}

Instrumentalisten geben signifikant häufiger als Nicht-Instrumentalisten an, Musik bewusst zu hören $\left(\mathrm{Chi}^{2}=10,0, d f=4, p=.03\right)$. Die Stimmungsregulierung scheint bei Instrumentalisten eine besondere Rolle zu spielen. Während sie der Aussage zustimmen, durch das Hören von Musik zufällig ihre Stimmung zu verbessern $\left(\mathrm{Chi}^{2}=9,4, d f=4, p=.034\right)$, geben sie gleichfalls an, dass Musikhören ihnen hilft, überschüssige Energien abzubauen $\left(\mathrm{Chi}^{2}=\right.$ $10,6, d f=4, p=.027)$ oder hierdurch neue Energien zu erhalten $\left(\mathrm{Chi}^{2}=\right.$ $15,3, d f=4, p=.001$ ). Als einzige Teilgruppe stimmen sie hoch signifikant häufiger der Aussage zu, dass sie Auftritte von Künstlern des von ihnen als 
potenziell Tabu brechend eingestuften Genres für obszön halten $\left(\mathrm{Chi}^{2}=\right.$ $13,1, d f=4, p=.006)$.

\subsection{Darstellung und Ergebnisse der Nebenstudie »Compilations«}

Ein Teilaspekt der quantitativen Studie hob darauf ab, persönliche Favoritenlisten der Vpn für die Bereiche »erotischer Song « und »erotische Künstlerin/erotischer Künstler « zu erfassen. Die Probanden nannten hierzu 83 Songs und 142 Künstler oder Interpreten (Mehrfachnennungen waren jeweils möglich). Es ergab sich daraus zunächst die folgende Rangliste für die erfassten Titel:

\begin{tabular}{|llc|}
\hline \multicolumn{1}{c}{ Interpret/Komponist } & \multicolumn{1}{c}{ Titel } & Nennungen (\%) \\
\hline 1. Jane Birkin \& Serge Gainsbourg & "Je t'aime... moi non plus & $5(6,0 \%)$ \\
Marvin Gaye & "Sexual Healing & $5(6,0 \%)$ \\
2. Marvin Gaye & "Let's Get It On« & $3(3,6 \%)$ \\
3. Maurice Ravel & "Boléro« & $2(2,4 \%)$ \\
\hline
\end{tabular}

Tabelle 1: Bestplatzierte Titel und Künstler der quantitativen Hauptstudie

Es erscheint in einer im Mittel vergleichsweise jungen Stichprobe erstaunlich, dass besonders Lieder der frühen 1970er und 1980er-Jahre - ein Großteil der Vpn war zu dieser Zeit noch nicht geboren oder maximal zehn Jahre alt - sowie ein kunstmusikalisches Werk die meisten Nennungen erfahren. Bei den weiteren 68 genannten Titeln handelt es sich ausschließlich um Einzelnennungen, die (bisher?) anscheinend nicht konsensfähig sind. Wenn also ein beginnender Traditionsstrom (vgl. Assmann 1992) für den Bereich »erotische Titel« innerhalb der Stichprobe beschrieben werden sollte, so läge dieser etwas später, als dies für die »allgemeine Kanonisierung « populärer Musik durch von Appen, Doehring und Rösing (2008: 30ff.) festgestellt wurde. Der hohe Grad der Differenzierung, besonders unter jungen Probanden, lässt die Vermutung zu, dass in diesem Alterssegment die nahezu entgrenzten Möglichkeiten, Musik zu finden, zu rezipieren, zu diskutieren, selbst zu produzieren oder zu verarbeiten, dem Traditionsstrom der Elterngeneration entgegenarbeiten, viele Nebenarme des Hauptstroms ausbilden und diesen weitläufig mäandern lassen.

Die Erfassung »erotischer Künstlerinnen/Künstler« förderte folgende Rangliste zu Tage: 


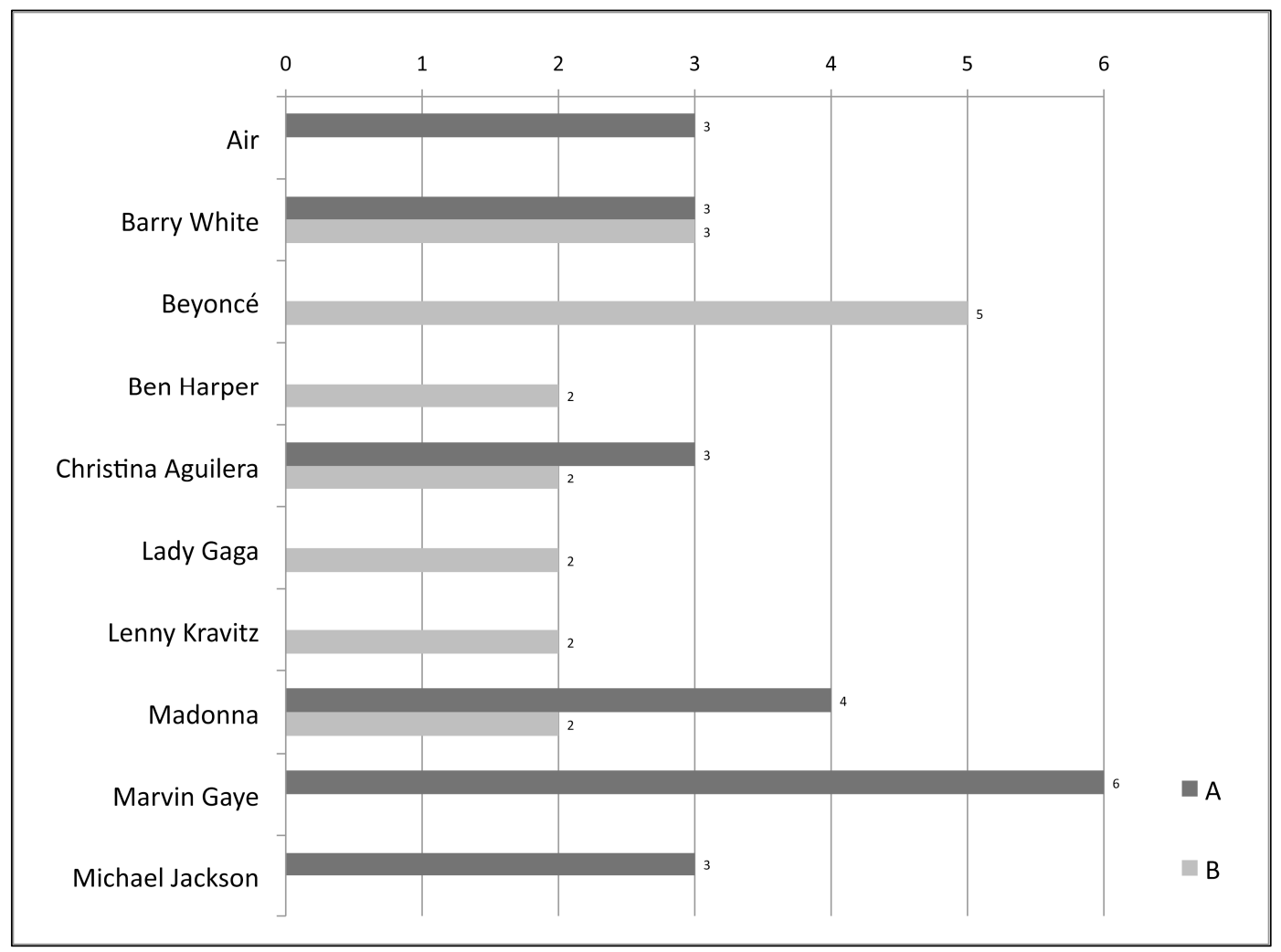

Abbildung 2: Häufigste Nennungen »erotischer Künstler« (A: Mit Liednennung, B: Ohne Liednennung)

Es wird an dieser Gegenüberstellung deutlich, dass beispielsweise im Fall von Marvin Gaye ausschließlich seine Musik für die Zuschreibung, er sei erotisch, verantwortlich zu machen ist. Im Fall der Interpretin Beyoncé wird hingegen nur ihre Person selbst in dieser Stichprobe für erotisch erachtet, keine der Vpn nannte einen Titel. Auch in dieser vergleichsweise kleinen Stichprobe werden Effekte der medialen Einflussnahme (vgl. Hickethier 2007, Schmidt 2005, Uhl 2009) auf die Präferenzbildung der Vpn sichtbar, betrachtet man bspw. die Nennungen von Lady Gaga oder Lenny Kravitz: Beide stehen für disparate Genres und Zielgruppen, dennoch besteht ein Großteil ihrer medialen Inszenierung und der dahinterstehenden Verkaufsstrategien aus einer bewussten Schwerpunktsetzung im Bereich Erotik. Diese noch als Tendenz oder Vermutung anzusehende These müsste in weiteren Studien überprüft werden.

Zeitgleich zur quantitativen Studie erfasste eine Teilstudie sämtliche zu einem Stichtag auf dem deutschen Markt verfügbaren Compilations, deren Namensgebung mit dem Forschungsthema assoziiert werden konnte. Hierzu zählen beispielsweise »Kuschelrock«, »Kuscheljazz« oder »Kuschelklassik«, »Erotic Lounge «, »Erotic Dreams«, »Sinners Lounge« und vergleichbare Veröffentlichungen. Hierdurch wurden insgesamt 3145 Angaben zu Songs und 2998 Angaben zu Interpretinnen/Interpreten bzw. Komponistinnen/Kompo- 
nisten erfasst, die nachfolgend ausgewertet und zu eigenen Rankings zusammengefasst wurden. Im Daten-Pool befinden sich neben PopmusikCompilations auch Werke aus Barock, Klassik, Romantik und Jazz, die an dieser Stelle bewusst mit Erwähnung finden sollen, um das Ziel zu unterstützen, die bisher von musikindustrieller Seite aus erfolgte Schwerpunktbildung im Bereich »erotisierende Musik« - zumindest für den deutschsprachigen Markt - transparenter werden zu lassen.

In der Auswertung des Compilations-Datenpools wird sehr gut deutlich, dass zwar einige vorab dort vermutete Titel wie beispielsweise »Je t'aime... moi non plus « von Jane Birkin und Serge Gainsbourg oder »Boléro « von Maurice Ravel unter den Bestplatzierten zu finden sind, bedingt durch die große Zahl verfügbarer Compilations aus dem House-, Trance- und ChilloutSegment aber auch vermeintlich unbekannte Titel mit auf den vorderen Rängen rangieren:

\begin{tabular}{|c|c|c|}
\hline Interpret/Komponist & Titel & Nennungen (\%) \\
\hline 1. Mo' Horizons & »Dance Naked Under Palmtrees« & $5(0,16 \%)$ \\
\hline Jazzamor & »Ain't No Sunshine « & $5(0,16 \%)$ \\
\hline Phil Collins & »In The Air Tonight « & $5(0,16 \%)$ \\
\hline Maurice Ravel & »Boléro« & $5(0,16 \%)$ \\
\hline 2. AKMusique & »Café Noir« & $4(0,13 \%)$ \\
\hline Piotr I. Tschaikowsky & »Der Schwanensee« & $4(0,13 \%)$ \\
\hline The Strike Boys & »Go Back Home« & $4(0,13 \%)$ \\
\hline Beatkonexion & $\gg$ Heart« & $4(0,13 \%)$ \\
\hline $\begin{array}{l}\text { Jane Birkin \& } \\
\text { Serge Gainsbourg }\end{array}$ & »Je t'aime... moi non plus« & $4(0,13 \%)$ \\
\hline Marvin Gaye & »Sexual Healing « & $4(0,13 \%)$ \\
\hline Brook Sappire & »So We Start« & $4(0,13 \%)$ \\
\hline Chris Isaak & »Wicked Game« & $4(0,13 \%)$ \\
\hline
\end{tabular}

Tabelle 2: Bestplatzierte Titel der Compilations-Studie $(\mathrm{N}=3144)$

Auch innerhalb dieser für den deutschen Tonträgermarkt im Jahr 2009 als repräsentativ zu bezeichnenden Studie ist auffällig, dass keiner der bestplatzierten Titel in der Lage ist, auch nur annähernd ein Prozent der gesamten Nennungen auf sich vereinen zu können. Ein deutlicher Kanon erotischer Songs ist also nicht zu identifizieren. Ebenso unerwartet liest sich die Liste der meistgenannten Künstlerinnen und Künstler: 


\begin{tabular}{|c|c|c|}
\hline & Interpret, Komponist & Nennung (\%) \\
\hline 1. & Vincenzo (\& Rasoul) & $20(0,67 \%)$ \\
\hline \multirow[t]{2}{*}{2.} & Gerhard Heinz & $19(0,63 \%)$ \\
\hline & Phil Collins & $19(0,63 \%)$ \\
\hline 3. & Johann Sebastian Bach & $18(0,60 \%)$ \\
\hline 4. & George Michael & $16(0,53 \%)$ \\
\hline 5. & Casellas Sextet Folk feat. Eva Trullas & $15(0,50 \%)$ \\
\hline 5. & Erotic Drum Band & $15(0,50 \%)$ \\
\hline 6. & Kevin Yost, Peter Funk & $14(0,46 \%)$ \\
\hline \multirow[t]{4}{*}{7.} & Celine Dion & $13(0,43 \%)$ \\
\hline & Jazzamor & $13(0,43 \%)$ \\
\hline & Prince & $13(0,43 \%)$ \\
\hline & Piotr I. Tschaikowsky & $13(0,43 \%)$ \\
\hline \multirow[t]{3}{*}{8.} & Mo' Horizons & $12(0,40 \%)$ \\
\hline & Movie Sound Orchestra feat. The L.A. Voices & $12(0,40 \%)$ \\
\hline & Toni Braxton & $12(0,40 \%)$ \\
\hline \multirow[t]{3}{*}{9.} & Levantis / Rajiv Basham Singh & $11(0,36 \%)$ \\
\hline & Mariah Carey & $11(0,36 \%)$ \\
\hline & Whitney Houston & $11(0,36 \%)$ \\
\hline \multirow[t]{4}{*}{10.} & Catchy Tongue & $10(0,33 \%)$ \\
\hline & Frédéric Chopin & $10(0,33 \%)$ \\
\hline & Simply Red & $10(0,33 \%)$ \\
\hline & Antonio Vivaldi & $10(0,33 \%)$ \\
\hline
\end{tabular}

Tabelle 3: Bestplatzierte Interpretinnen/Interpreten bzw. Komponistinnen/Komponisten der Compilations-Studie $(\mathrm{N}=2998)$

Interessant hieran ist beispielsweise der bestplatzierte Vincenzo: ein Hamburger Projekt um den Produzenten und DJ Vincenzo Ragone, der es auf 20 Nennungen bringt, die jeweils aber nur zwei oder dreimal innerhalb der Titelliste Erwähnung finden. Anscheinend hat sich im Gebiet der Erotik ein Teilmarkt für funktionale Musik etablieren können, der im Stande ist, über eine bewusste Genre-Auswahl unterschiedliche Rezipientengruppen anzusprechen. Um potenzielle Strategien der Produktmanager und Verantwortlichen für die Compilations nachzuvollziehen, wurde die These formuliert, dass eine Vielzahl der zusammengefassten Lieder lediglich auf der Grundlage der im Titel genannten Wortkombinationen mit aufgenommen wurden. Dieses Vorhaben wurde durch eine quantitative Auswertung des Datenpools über die Erfassung von Schlagworten bzw. deren Bestandteilen realisiert: 


\begin{tabular}{|lcc|}
\hline Schlagwort & Anzahl & $\begin{array}{c}\text { Anteil an der } \\
\text { Titelauswahl in \% }\end{array}$ \\
\hline "lov* & 314 & $9,98 \%$ \\
"rom* & 77 & $2,45 \%$ \\
"heart* & 43 & $1,37 \%$ \\
"sex* & 34 & $1,08 \%$ \\
"feel* & 33 & $1,05 \%$ \\
"body* & 30 & $0,95 \%$ \\
"erotic* & 12 & $0,38 \%$ \\
"amor* & 12 & $0,38 \%$ \\
"oh* & 9 & $0,29 \%$ \\
"fuck* & 8 & $0,25 \%$ \\
"lieb* & 6 & $0,19 \%$ \\
"hurt* & 5 & $0,16 \%$ \\
"puss* & 4 & $0,13 \%$ \\
"horny* & 3 & $0,10 \%$ \\
\hline
\end{tabular}

Tabelle 4: Schlagwörter in der Titelauswahl $(\mathrm{N}=3145)$

Man sieht sehr gut, dass Songs, die den Teilbegriff »*lov* «einhalten, mit annähernd $10 \%$ aller Nennungen, mit vergleichsweise hoher Wahrscheinlichkeit auf derartigen Compilations zu finden sind. Weiterhin finden sich Worte wie »Romanze«, »romantic « in 77 und »heart « in 43 Titeln. Explizite Begriffe hingegen wie »*fuck* « oder »*horny*« finden sich eher selten. Der Großteil des erfassten Materials besteht aus englischsprachigen Titeln, vereinzelt finden sich spanische Songs; deutsche Titel stammen vorwiegend aus dem Bereich der Kunstmusik. Ein vertiefender Forschungsansatz würde hier versuchen, den erfassten Titel durch mehrere Rater, unterteilt nach ihrem Expertisegrad, Kategorien und musikalischen Genres zuordnen zu lassen, um so zu quantifizierbaren und dezidierten Aussagen über die materiale Struktur des Datenpools kommen zu können. Qualitative Interviews mit den für die Produktlinien Verantwortlichen wären eine weitere methodische Vorgehensweise, um die Entstehung dieser Tonträger-Reihen plastischer werden zu lassen.

\section{Fazit und Schlussbetrachtung}

Aus dem noch wenig übersichtlichen, geschweige denn geordneten Bereich der Forschungen zu Popmusik, Medien und Emotionen haben wir hinsichtlich der Auswahl und des Einsatzes von Musik für erotische und sexuelle Kontexte mögliche Forschungsperspektiven erarbeiten können. Anhand der von 
uns durchgeführten explorativen Hauptstudie sowie der Nebenstudie (Compilations) wurden folgende Tendenzen deutlich:

- Popmusik wird im alltäglichen Gebrauch häufig bewusst gehört. Sie nimmt im Leben vieler Probanden eine wichtige Stellung ein (Dauer und Nutzung).

- Die Teilnehmenden versuchen zum überwiegenden Teil hierdurch, ihre subjektive Stimmung zu verbessern (Stimmungsregulierung/Kompensationsprinzip).

- Während für die Entstehung erotischer und sexueller Situationen nach Aussage der Probanden ihre Stimmung, ihr Partner und der Faktor Zeit von empfundener Relevanz sind, zählen auf Seiten der musikalischen Parameter das Tempo sowie die Stimme des Interpreten zu den meist genannten Angaben (Bedingungen für Erotik/musikalische Parameter).

- Die Stichprobe ist sich hinsichtlich der Fähigkeit zur Erotisierung und Sexualisierung von zwischenmenschlichen Momenten weitgehend einig, dass Genres wie Soul, Lounge oder R\&B eher als unterstützend empfunden werden, Heavy Metal, Schlager und HipHop hingegen eher als störend (Kompatibilität von Popmusik-Genre und Erotisierung/Sexualisierung).

- Drei Viertel der Versuchspersonen sind nach eigener Meinung in der Lage, über die Musikauswahl eine erotische Stimmung zu erzeugen und wählen dabei vorwiegend Stücke aus, die sie ursprünglich in einem erotischen oder sexuellen Kontext wahrgenommen haben (Auswahl/Beziehungen/Rituale).

- Während der Anbahnung erotischer Handlungen hört der überwiegende Teil der Befragten gerne Popmusik. In dieser Studie wird Musik auch vergleichsweise häufiger, als in bisherigen Studien nachgewiesen werden konnte, während des sexuellen Aktes akzeptiert (Aktionen und Handlungen).

- Die Musik an sich ist auch im Spiegel der vorliegenden Ergebnisse als selbstreferenzieller Bereich nicht in der Lage, aus sich heraus Tabus zu brechen oder pornographisch zu sein. Dahingegen werden vor allem in den Genres Rap und Heavy Metal die Lyrics als Hauptursache für derlei Zuschreibungen identifiziert (Porno/Tabu).

Erste Effekte, die einer statistischen Belastung standhalten, konnten weiterhin für die soziodemographischen Faktoren Gender, Beruf und instrumentale Vorkenntnisse beschrieben werden. Darüber hinaus zeigt die erste Erschließung des Datenmaterials individueller und kommerzieller Liedzusammenstellungen für die Anbahnung und Durchführung sexueller Handlun- 
gen, dass hierbei sowohl die Musiker selbst (Person/Image) als auch Vermarktungsstrategien und mediale Präsentationsformen und Rahmungen einer weiteren Aufschlüsselung bedürfen. Es lässt sich dennoch bereits jetzt sagen, dass unter jungen Rezipienten anscheinend eine Abkehr vom bisherigen Traditionsstrom erotischer Popmusik hin zu einer stärker individualisierten und an persönlichen, situativen Kontexten orientierten Auswahl einsetzt. Dieser Trend lässt sich derzeit vorwiegend qualitativ beobachten.

Als Forschungsrückblick verbliebe zunächst die Forderung, die benutzten theoretischen Vorlagen noch deutlicher integrativ auf den hoch spezifischen Zusammenhang von Popmusik und Erotik auszurichten. Weiterhin kann eine Aufnahme der persönlichen sexuellen Dispositionen zu einem verbesserten Verständnis beitragen. Die vorliegende Pilotstudie verbleibt zunächst auf Variablenebene, eine Optimierung der Skalen würde es für Folgestudien ermöglichen, auch auf Ebene der Konstrukte weitergehende, sprich inferenzstatistisch gesicherte Aussagen treffen zu können. Diese Zuspitzung könnte den vermeintlichen Widerspruch zwischen kaum vorhandener Fachliteratur und demgegenüber Brisanz sowie Relevanz des Themas auflösen und zu einer weiteren Etablierung von Erforschungen dieses komplexen Themenzusammenhangs führen, wie er pointiert von Jeffrey J. Arnett formuliert wurde: »Popular music and sex have gone together like a horse and carriage ever since the days of the horse and carriage « (Arnett 2002: 254).

\section{Literaturverzeichnis}

Appen, Ralf von / Doehring, André / Rösing, Helmut (2008). »Pop zwischen Historismus und Geschichtslosigkeit. Kanonbildung in der populären Musik. «In: No time for loser. Charts, Listen und andere Kanonisierungen in der populären Musik. Hg. v. Dietrich Helms und Thomas Phleps (= Beiträge zur Popularmusikforschung 36). Bielefeld: transcript, S. 25-49.

Arnett, Jeffrey Jensen (2002). "The Sounds of Sex: Sex in Teens' Music and Music Videos."In: Sexual Teens, Sexual Media: Investigating Media's Influence on Adolescent Sexuality. Hg. v. Jane D. Brown, Jeanne R. Steele und Kim WalshChilders. Mahwah: Erlbaum, S. 253-264.

Assmann, Jan (1992). Das kulturelle Gedächtnis. Schrift, Erinnerung und politische Identität in frühen Hochkulturen. München: Beck.

Ben-Ze'ev, Aaron (2009). Die Logik der Gefühle. Kritik der emotionalen Intelligenz. Frankfurt/M.: Suhrkamp.

Bortz, Jürgen (2005). Statistik für Human- und Sozialwissenschaftler. Heidelberg: Springer (6. Aufl.).

Bortz, Jürgen / Döring, Nicola (2006). Forschungsmethoden und Evaluation: für Human- und Sozialwissenschaftler. Berlin u.a.: Springer (4. Aufl.).

Bühl, Walter Ludwig (2004). Musiksoziologie. Frankfurt/M. u.a.: Peter Lang. 
Bühner, Markus (2004). Einführung in die Test- und Fragebogenkonstruktion. München u.a.: Pearson Studium.

Büsser, Martin / Behrens, Roger / Engelmann, Jonas / Ullmaier, Johannes (Hg.) (2008). testcard - Beiträge zur Popgeschichte, H. 17: Sex. Mainz: Ventil.

Casimir, Torsten (1991). Musikkommunikation und ihre Wirkungen. Eine systemtheoretische Kritik. Wiesbaden: DUV.

DeNora, Tia (1997). »Music and Erotic Agency - Sonic Resources and Social-Sexual Action.«In: Body \& Society 3, H. 2, S. 43-65.

DeNora, Tia (1999). »Music as a Technology of the Self.«In: Poetics. Journal of Empirical Research on Culture, the Media and the Arts, H. 27, S. 31-56.

DeNora, Tia (2001). "Aesthetic Agency and Musical Practice: New Directions in the Sociology of Music and Emotion. "In: Music and Emotion. Theory and Research. Hg. v. Patrick N. Juslin und John A. Sloboda. Oxford: University Press, S. 161180.

Diederichsen, Diedrich (1985). »Sexbeat: Jesus und Maria.« In: Das Abenteuer Liebe. Bestandsaufnahme eines unordentlichen Gefühls. Hg. v. Peter Kemper und Ulrich Sonnenschein. Frankfurt/M.: Suhrkamp, S. 74-80.

Diederichsen, Diedrich (2006). »Indie im Kampf mit dem Index. Über das Verhältnis von Pornographie und Popkultur.«In: Texte zur Kunst 64: Porno. Hg. v. Isabelle Graw. Berlin: Texte zur Kunst, S. 37-47.

Dollase, Rainer / Rüsenberg, Michael / Stollenwerk, Hans J. (1974). Rock People oder Die befragte Szene. Frankfurt/M.: Fischer.

Dollase, Rainer / Rüsenberg, Michael / Stollenwerk, Hans J. (1985). »Kohortenspezifische Sozialisation. «In: Musikpsychologie. Ein Handbuch in Schlüsselbegriffen. Hg. v. Herbert Bruhn, Rolf Oerter und Helmut Rösing. München: Urban und Schwarzenberg, S. 204-209.

Eismann, Sonja (Hg.) (2007). Hot Topic. Popfeminismus heute. Mainz: Ventil.

Flick, Uwe (2009). Qualitative Sozialforschung: eine Einführung. Reinbek bei Hamburg: Rowohlt (2. Aufl.).

Fritz, Irina / Klingler, Walter (2006). »Medienzeitbudgets und Tagesablaufverhalten. Ergebnisse auf Basis der ARD/ZDF-Studie Massenkommunikation 2005. «In: Media Perspektiven, H. 4, S. 222-234.

Gabrielsson, Alf / Lindström, Erik (2001). »The Influence of Musical Structure on Emotional Expression. «In: Music and Emotion. Theory and Research. Hg. v. Patrik N. Juslin und John A. Sloboda. Oxford: Oxford University Press, S. 223-247.

Gries, Rainer (2006). »Das generationengeschichtliche Paradigma in der Kommunikationshistorie. «In: Medien \& Zeit. Kommunikation in Vergangenheit und Gegenwart 21, H. 3, S. 4-20.

Großmann, Rolf (1991). Musik als >Kommunikation<. Zur Theorie musikalischer Kommunikationshandlungen. Braunschweig: Vieweg.

Helfferich, Cornelia (2005). Die Qualität qualitativer Daten. Wiesbaden: Verlag für Sozialwissenschaften (2. Aufl.).

Helms, Dietrich (2009). »Amor docet musicam - Zu Theorie und Geschichte eines intimen Verhältnisses. «In: Musik - Geschichte(n) - Erzählen. Freundesgabe für Hans-Christian Schmidt-Banse zur Emeritierung. Hg. v. Hartmuth Kinzler. Osnabrück: Epos, S. 79-94.

Hickethier, Knut (2007). »Die kulturelle Bedeutung medialer Emotionserzeugung.« In: Audiovisuelle Emotionen. Emotionsdarstellung und Emotionsvermittlung durch audiovisuelle Medienangebote. Hg. v. Anne Bartsch, Jens Eder und Kathrin Fahlenbrach. Köln: von Halem, S. 104-122. 
Jacke, Christoph (2008). „Keine Musik ohne Medien, keine Medien ohne Musik? Pop(-kulturwissenschaft) aus medienwissenschaftlicher Perspektive.« In: PopMusicology. Perspektiven der Popmusikwissenschaft. Hg. v. Christian Bielefeldt, Udo Dahmen und Rolf Grossmann. Bielefeld: transcript, S. 135-152.

Jacke, Christoph (2009). Einführung in Populäre Musik und Medien. Münster u.a.: LIT.

Jacke, Christoph / Meinecke, Thomas (2008). »Vorübergehende Vergegenwärtigungen in der Popkultur. Ein Gespräch über das Sprechen über und das Erinnern von Pop. «In: Populäre Kultur und soziales Gedächtnis: theoretische und exemplarische Überlegungen zur dauervergesslichen Erinnerungsmaschine Pop. Popular Culture and Social Memory: Theoretical and Empirical Analyses on The Oblivious $>$ Memory-Machine $<$ Pop. Hg. v. Christoph Jacke und Martin Zierold (= Siegener Periodicum zur Internationalen Empirischen Literaturwissenschaft 24/2). Frankfurt/M. u.a.: Peter Lang, S. 239-256.

Jacke, Christoph / Zierold, Martin (2008). »Pop - die vergessliche Erinnerungsmaschine. "In: Populäre Kultur und soziales Gedächtnis. Theoretische und exemplarische Überlegungen zur dauervergesslichen Erinnerungsmaschine Pop. Popular Culture and Social Memory: Theoretical and Empirical Analyses on The Oblivious >Memory-Machine Pop. Hg. v. Christoph Jacke und Martin Zierold (= Siegener Periodicum zur Internationalen Empirischen Literaturwissenschaft 24/2). Frankfurt/M. u.a.: Peter Lang, S. 199-210.

Jacke, Christoph / Zierold, Martin (2009). »Produktive Konfrontationen. Warum der Erinnerungsdiskurs von dem Austausch mit der Popkulturforschung profitiert und umgekehrt. «In: Medien \& Zeit. Kommunikation in Vergangenheit und Gegenwart 24, H. 4, S. 4-13.

Juslin, Patrik N / Sloboda, John A. (Hg.) (2001). Music and Emotion. Theory and Research. Oxford: Oxford University Press.

Juslin, Patrick N. / Sloboda, John A. (2001a). »Music and Emotion: Commentary.« In: Music and Emotion. Theory and Research. Hg. v. Patrick N. Juslin und John A. Sloboda. Oxford: Oxford University Press, S. 453-462.

Kemper, Peter (2005). "Love goes Pop. Die lärmende Macht großer Gefühle.« In: Liebe - Zwischen Sehnsucht und Simulation. Hg. v. Peter Kemper und Ulrich Sonnenschein. Frankfurt/M.: Suhrkamp, S. 297-306.

Kim, Ji-Hun (2009). »Nach Google. Eyeplorer \& Wolfram Alpha. Interview mit dem Heisenberg-Enkel und Eyplorer-Gründer Martin Hirsch.« In: De:Bug. Elektronische Lebensaspekte. Magazin für Musik, Medien, Kultur, Selbstbeherrschung, H. 134, S. 22-24.

Korte, Hermann (2008). „Was heißt: >Das bleibt<? Bausteine zu einer kulturwissenschaftlichen Kanontheorie. "In: No time for losers. Charts, Listen und andere Kanonisierungen in der populären Musik (= Beiträge zur Popularmusikforschung 36). Hg. v. Dietrich Helms und Thomas Phleps. Bielefeld: transcript, S. 11-23.

Kreutz, Gunter (1997). »Musikrezeption zwischen >Liebestraum< und >Love-Parade. Sexualität und Sinnlichkeit im Erleben von Musik. «In: Zeitschrift für Medienpsychologie 9, H. 4, S. 293-311.

Kreutz, Gunter (1999). "Gender Differences and Sociographical Factors of Erotic Signification in Music." In: Music and Signs. Semiotic and Cognitive Studies in Music. Hg. v. Ioannis Zannos. Bratislava: ASCO Art and Science, S. 393-407.

Kreutz, Gunter (2002). »Manche mögen's heiß... Über die erotische Sinnlichkeit der Musik und des Musikerlebens. "In: Musik und Unterricht, H. 67, S. 32-38. 
Kreutz, Gunter (2008). »Musik und Emotion.« In: Musikpsychologie. Das neue Handbuch. Hg. v. Herbert Bruhn, Reinhard Kopiez und Andreas Lehmann. Reinbek bei Hamburg: Rowohlt Taschenbuch, S. 548-572.

Lehmann, Andreas C. (1994). Habituelle und situative Rezeptionsweisen beim Musikhören. Eine einstellungstheoretische Untersuchung. Frankfurt/M. u.a.: Peter Lang.

Luhmann, Niklas (1994). Liebe als Passion. Zur Codierung von Intimität. Frankfurt/M.: Suhrkamp.

Luhmann, Niklas (2008). Liebe. Eine Übung. Frankfurt/M.: Suhrkamp.

Mayring, Philipp (2008). Qualitative Inhaltsanalyse. Weinheim u.a.: Beltz (10. Aufl.).

Medienpädagogischer Forschungsverbund Südwest (2008). Jugend, Information, (Multi-)Media. Basisstudie zum Medienumgang 12- bis 19-Jähriger in Deutschland. Stuttgart: Landesanstalt für Kommunikation Baden-Württemberg.

Meier, Andreas (2009). Tabubrüche in der Musik. Über den Zusammenhang zwischen gezielten Tabubrüchen und dem Käuferverhalten in der Musikindustrie. Münster: Telos Verlag.

Merten, Klaus (1999). Einführung in die Kommunikationswissenschaft. Bd. 1: Grundlagen der Kommunikationswissenschaft. Münster u.a.: LIT.

Meyer, Leonard B. (2001). Music and Emotion: Distinctions and Uncertainties. In: Music and Emotion. Theory and Research. Hg. v. Patrik N. Juslin und John A. Sloboda. Oxford: Oxford University Press, S. 341-360.

Pfleiderer, Martin (2009). "Stimmen populärer Musik. Vokale Gestaltungsmittel und Aspekte der Rezeption. «In: Hamburger Jahrbuch für Musikwissenschaft 25. Hg. v. Rolf Bader und Friedrich Geiger. Frankfurt/M. u.a.: Peter Lang, S. 233-274.

Pressetext RTL (2009). "Die ultimative Chart Show«. Programmankündigung http://www.rtl.de/cms/unterhaltung/tv-programm/show/die-ultimativechartshow.html (Zugriff am 29.5.2009).

Reitsamer, Rosa / Weinzierl, Rupert (Hg.) (2006). Female Consequences. Feminismus, Antirassismus, Popmusik. Wien: Erhard Löcker.

Roth, Gerhard (1996). »Erkenntnis und Realität. Das reale Gehirn und seine Wirklichkeit.« In: Der Diskurs des Radikalen Konstruktivismus. Hg. v. Siegfried J. Schmidt. Frankfurt/M.: Suhrkamp (7. Aufl.), S. 229-255.

Roth, Gerhard (2003). »Verstand oder Gefühl - auf was sollen wir hören? « In: Aus Sicht des Gehirns. Hg. v. dems. Frankfurt/M.: Suhrkamp, S. 154-165.

Roth, Gerhard (2004). "Sexualität, Verliebtsein und Liebe."In: Das Abenteuer Liebe. Bestandsaufnahme eines unordentlichen Gefühls. Hg. v. Peter Kemper und Ulrich Sonnenschein. Frankfurt am Main: Suhrkamp, S. 219-228.

Roth, Gerhard (2007). Fühlen, Denken, Handeln. Frankfurt/M.: Suhrkamp.

Schenk, Michael (1987). Medienwirkungsforschung. Tübingen: Mohr.

Schmidt, Siegfried J. (2005). »Medien und Emotionen. Zum Management von Bezugnahmen.«In: Medien und Emotionen. Hg. v. Siegfried J. Schmidt. Münster: LIT, S. 11-39.

Schramm, Holger (2005). Mood Management durch Musik. Die alltägliche Nutzung von Musik zur Regulierung von Stimmungen. Köln: von Halem.

Schramm, Holger (Hg.) (2009). Handbuch Musik und Medien. Konstanz: UVK.

Schwender, Clemens / Schwab, Frank (2007). »Evolutionäre Grundlagen emotionaler und ästhetischer Medienrezeption. «In: Audiovisuelle Emotionen. Emotionsdarstellung und Emotionsvermittlung durch audiovisuelle Medienangebote. Hg. v. Anne Bartsch, Jens Eder und Kathrin Fahlenbrach. Köln: von Halem, S. 60-84. 
Seim, Roland / Spiegel, Josef (2004). Nur für Erwachsene. Rock und Popmusik: zensiert, diskutiert und unterschlagen. Münster: Telos Verlag.

Silverstone, Roger (2007). Anatomie der Massenmedien. Ein Manifest. Frankfurt/M.: Suhrkamp.

Singer, Wolf (2006). Vom Gehirn zum Bewusstsein. Frankfurt/M.: Suhrkamp.

Sloboda, John A. / Lamont, Alexandra; Greasly, Alinka (2009). "Choosing to Hear Music. Motivation, Process, and Effect.« In: The Oxford Handbook of Music Psychology. Hg. v. Susan Hallam, Ian Cross und Michael Thaut. Oxford: Oxford University Press, S. 431-440.

Sloboda, John A. / O'Neill, Susan A. (2001). „Emotions in Everyday Listening to Music. «In: Music and Emotion. Theory and Research. Hg. v. Patrik N. Juslin und John A., Sloboda. Oxford: Oxford University Press, S. 415-429.

Uhl, Matthias (2009). Medien - Gehirn - Evolution. Mensch und Medienkultur verstehen. Eine transdisziplinäre Medienanthropologie. Bielefeld: transcript.

Verbrauchs- und Medienanalyse (2010). Basisauswertung. Online unter: http:// www.vuma.de/de/downloads.html (Zugriff am 22.6.2010)

Wulf, Carmen (2008). Historischer Wandel von Liebesvorstellungen. Theoretische Aspekte emotionalen Wandels und empirische Untersuchung des Wandels von Liebesauffassungen in populären Liebeliedern. Hamburg: Dr. Kovac.

Zierold, Martin (2006). Gesellschaftliche Erinnerung. Eine medienkulturwissenschaftliche Perspektive. Berlin: de Gruyter.

Zöfel, Peter (2008). Statistik für Psychologen im Klartext. München u.a.: Pearson Studium.

\begin{abstract}
This paper provides a short review of literature in the field of popular music, erotic/sexual emotions and media on the one hand and, on the other hand, it asks some crucial questions about the choice and use of popular music in erotic situations. These questions are answered by an empirical two-part study. New data are presented which consider the nature of music used in erotic situations, including the genres of music suitable for such contexts, the musical parameters of such music, the ways in which and reasons why people use music in erotic and sexual contexts, the rituals and aspects of relationships that may influence music selection, and the notion of pornographic music.
\end{abstract}

